\title{
Implementation of a brief anxiety assessment and evaluation in a Department of Veterans Affairs geriatric primary care clinic
}

\author{
Christine E. Gould, PhD; ${ }^{1-2 *}$ Sherry A. Beaudreau, PhD, ABPP; ${ }^{2-3}$ Gail Gullickson, MD; ${ }^{1,4}$ J. Lisa Tenover, MD, \\ PhD; ${ }^{1,4}$ Elizabeth A. Bauer, RN, MS, AGCNS-BC; ${ }^{1}$ J. W. Terri Huh, PhD, ABPP ${ }^{1-2}$ \\ ${ }^{1}$ Geriatric Research, Education and Clinical Center, Department of Veterans Affairs (VA) Palo Alto Health Care Sys- \\ tem, Palo Alto, CA; ${ }^{2}$ Department of Psychiatry and Behavioral Sciences, Stanford University School of Medicine, Stan- \\ ford, CA; ${ }^{3}$ Sierra Pacific Mental Illness Research, Education and Clinical Center, VA Palo Alto Health Care System, \\ Palo Alto, CA; and School of Psychology, University of Queensland, Brisbane, Australia; ${ }^{4}$ Department of Medicine, \\ Stanford University School of Medicine, Stanford, CA
}

\begin{abstract}
Anxiety disorders are common and debilitating in older individuals, yet anxiety is often not formally assessed in primary care. We conducted a quality improvement project to examine the feasibility of implementing a brief anxiety assessment, the Geriatric Anxiety Inventory (GAI), in a Department of Veterans Affairs geriatric primary care clinic. We compared the GAI with a depression assessment, the 15-item Geriatric Depression Scale (GDS-15). Fifty older Veterans (mean age $=78.5+/-7.4$ yr) completed the GAI and GDS-15. Mean completion time and feedback to patients was brief (6.20 min; $n=10)$. Good internal consistency (alpha $=0.82$ ) was found for GAI scores. Patients with psychiatric diagnoses obtained significantly higher GAI scores (mean $=4.73+/-1.15$ ) compared with patients without psychiatric diagnoses (mean $=1.15+/-1.86, t(11.46)=-3.10$, $p=0.01$ ). Findings suggest that the GAI is acceptable to patients but may not be suitable for differentiating anxiety symptoms or disorders from depression. Interdisciplinary team members continued to implement the GAI after project completion to screen for and track anxiety symptoms in our geriatric primary care patients. Detecting anxiety with the GAI had the benefit of allowing providers to initiate conversations about available treatments and track symptoms as part of noting treatment progress.
\end{abstract}

Key words: anxiety disorder, anxious, depression, mental health, older adults, program evaluation, psychiatric, quality improvement, screening, Veteran.

\section{INTRODUCTION}

Anxiety disorders are the most common type of psychiatric illness [1], affecting one in three Vietnam Veterans exposed to war zone stress [2]. Vietnam Veterans are twice as likely to have elevated anxiety symptoms than World War II and Korean war era Veterans [3]. The presence of anxiety disorders and elevated anxiety symptoms contributes to negative physical and mental health outcomes, including elevated risk of myocardial infarctions [4], lower

\footnotetext{
Abbreviations: BAI = Beck Anxiety Inventory, GAD = generalized anxiety disorder, GAD-7 = Generalized Anxiety Disorder 7-item Scale, GAI = Geriatric Anxiety Inventory, GAI-SF = GAI short form, GDS-15 = 15-item Geriatric Depression Scale, KR20 = Kuder-Richardson 20 coefficient, MMSE = Mini-Mental State Examination, MOCA = Montreal Cognitive Assessment, PHQ-2 = two-item Patient's Health Questionnaire, QI = quality improvement, SBT = Short Blessed Test, SD = standard deviation, VA = Department of Veterans Affairs, VHA = Veterans Health Administration.

*Address correspondence to Christine E. Gould, PhD; VA Palo Alto Health Care System, GRECC, 3801 Miranda Ave (182B), Palo Alto, CA 94304; 650-493-5000, ext 6889; fax: 650-496-2505. Email: Christine.Gould@va.gov http://dx.doi.org/10.1682/JRRD.2014.10.0258
} 
cognitive functioning [5], and suicidal ideation [6]. Moreover, elevated anxiety symptoms precede accelerated functional decline in older adults [7].

Most older adults receive mental health treatment in primary care rather than mental health care clinics [8], yet anxiety disorders often are unrecognized in this setting [9]. Antidepressants, primarily SSRIs (selective serotonin reuptake inhibitors), are the main pharmacological line of treatment for late-life anxiety disorders [10]. Efficacious psychological treatments for anxiety disorders [11] and psychotherapy to augment pharmacotherapy [12] also exist but may not be offered to all patients due to the limited number of qualified providers. However, without adequate recognition of anxiety disorders, older adults do not receive any treatment for these issues.

When assessing older adults for anxiety and other issues, the clinician faces several challenges that may not be present with middle aged or young adults. The clinician must consider the individual's cognitive functioning, because cognitive impairment is associated with reduced reliability and validity on measures of psychiatric symptoms. Also, if the older adult is experiencing somatic symptoms related to a chronic medical condition or acute illness, the presence of these symptoms may confound reporting of anxiety symptoms on measures that include multiple somatic symptoms, such as the Beck Anxiety Inventory (BAI) [13]. As a further challenge, most anxiety screening guidelines or reviews [14] do not provide recommendations specifically for older adults. One exception to this is the Substance Abuse and Mental Health Services Administration's and the Administration on Aging's recommendation to use the Generalized Anxiety Disorder 7-item scale (GAD-7) [15] to screen for anxiety [16]. However, the GAD-7 has not been examined specifically in older adults and is limited to screening for generalized anxiety disorder (GAD) but not other common anxiety disorders (e.g., panic disorder, social phobia). Furthermore, the GAD-7 uses a four-point Likert-type scale, which may be confusing for some patients with cognitive impairment and visual memory deficits in particular [17].

To address the need for assessment guidelines specifically for older Veterans, Gould et al. conducted a systematic review that identified eight self-report anxiety measures that have psychometric support in older adults and are feasible to use in Department of Veterans Affairs (VA) primary care clinics [13]. The Geriatric Anxiety Inventory (GAI) [18] was identified as the best overall anxiety screen for older VA patients due to the strong psy- chometric support and the clear administration and scoring instructions. The GAI is a 20-item, dichotomously scored measure that can be administered in less than $3 \mathrm{~min}$. It assesses generalized anxiety symptoms, yet also has been validated to identify any anxiety disorder [19]. In a recent study with individuals with cognitive impairment and dementia, the GAI was examined and found to have excellent internal consistency in those with cognitive impairment and those with dementia $(\alpha=0.92,0.91)$ [20].

We conducted a quality improvement (QI) project with the aim of testing the feasibility of implementing the GAI to screen for anxiety in a VA geriatric primary care clinic. Establishing the feasibility of administering valid brief assessments for anxiety to older Veterans is imperative, particularly in light of the scope of the issue of anxiety in the aging population of Vietnam Veterans. Here, we report on the feasibility and process of implementing the GAI, the acceptability of the measure to VA patients and staff, and the prevalence of anxiety in this clinic using GAI scores. We also compared the GAI with the 15-item Geriatric Depression Scale (GDS-15) [21], since depression and anxiety are often comorbid [22]. The GDS-15 is used in our geriatrics clinic to assess depression in addition to the two-item Patient's Health Questionnaire (PHQ-2), an annual depression screen in the Veterans Health Administration (VHA). The goal of this project was to conduct an evaluation of a clinical care practice, namely the utility of integrating a brief anxiety assessment as part of routine practice in a geriatric primary care clinic. We were also interested in determining whether assessing anxiety with the GAI would provide added benefit to patients above and beyond a depression screen.

\section{METHODS}

\section{Participants}

Participants were 50 Veterans who attended a VA geriatrics primary care outpatient clinic between September 2012 and July 2013. Exclusion criteria included a diagnosis of dementia in the medical record or impairment on brief cognitive assessments (Mini-Mental State Examination [MMSE], Montreal Cognitive Assessment [MOCA], and Mini-Cog) completed within a year prior to the patient's visit. The selection of patients is described in greater detail in the "Procedure" section. 


\section{Measures}

The GAI is a 20-item measure of anxiety that uses a dichotomous "agree” or "disagree” response scale [18]. Scores range from 0 to 20, with higher scores indicative of greater anxiety severity. Findings from previous studies provide evidence for excellent GAI score test-retest reliability ( $r=0.93$ and 0.91 ) and excellent internal consistency $[18,23]$. We examined the psychometric properties of the GAI and found good internal consistency as measured with the Kuder-Richardson 20 coefficient (KR-20 = 0.82 ). Additional studies provide evidence of convergent validity [18,23-24] and discriminant validity [18].

The short GDS-15 [21] was used to measure depressive symptoms. The GDS-15 employs a dichotomous "yes" or "no" response scale. GDS-15 scores range from 0 to 15 , with higher scores indicative of greater depression severity. Studies provide support for internal consistency, reliability, and convergent validity with other measures of depression and discriminant validity [25]. Adequate internal consistency was found for the GDS-15 (KR-20 = 0.67) in the present sample of older VA patients.

We selected the Short Blessed Test (SBT) [26], a brief version of the Blessed Information-Memory-Concentration Test [27], as the brief cognitive assessment to identify and exclude patients with significant (SBT > 4) cognitive impairment [28]. The SBT is shorter than other cognitive assessments, such as the MMSE [29] and the MOCA [30]. The SBT also can be administered to individuals with impaired vision or those who have difficulty writing or drawing. Higher scores on the SBT indicate more errors. We gathered information on psychiatric diagnoses from the problem list in each patient's medical record.

\section{Procedure}

The VA Office of Research and Development reviewed the project prior to its implementation and granted it exempt status as a QI program.

Several steps were taken to select patients for the QI project. A psychology postdoctoral fellow (C.E.G.) reviewed the medical records of 131 older patients scheduled to attend the clinic. We excluded 23 patients with a dementia diagnosis on their problem list because of the questionable validity of self-report measures of anxiety and mood in individuals with dementia [31]. We excluded 11 patients identified as having "possible cognitive impairment or dementia" based on the results of one of three brief cognitive assessment used in the clinic within the last year: Mini-Cog ( $\geq 3$ ) [32], MMSE (>1.5 standard deviation [SD] below the mean for age and education) [29], or MOCA ( $>1.5$ SD below the mean for age and education) [30]. Thus, the remaining 97 patients were eligible because they either had (1) no dementia diagnosis and no brief cognitive assessment completed within a year of their upcoming primary care appointment or (2) no dementia diagnosis and a completed cognitive assessment with findings of no impairment within a year of their upcoming primary care appointment. Eligible patients were discussed during primary care team meetings with the attending physicians to determine whether any acute medical issues were present that required immediate attention such as hospitalization. Two patients with acute medical issues and one patient who did not speak English were excluded. One additional patient was excluded based on the attending physician's (G.G.) recommendation given the patient's strong concerns with privacy issues in the past.

Eligible patients were approached during their regular primary care visit. An attending physician (G.G., J.L.T.) first introduced the psychology fellow to the patients, and then the fellow (C.E.G.) explained the nature of the project to the patients. She explained to the patients that these assessments are similar to the questions that they are asked on an annual basis about their mood (e.g., PHQ-2). Patients were informed that stress and anxiety are common but are often not asked about formally during primary care visits. The psychology fellow stated that the aim of the project was to see if this measure, the GAI, was a useful way of asking about anxiety and stress. She communicated that choosing not to participate in the QI project would in no way affect the care they already receive or will receive in the future through the VA. If a patient agreed to participate in the project, a brief cognitive assessment (SBT) was administered if no cognitive assessment (Mini-Cog, MMSE, or MOCA) had been administered in the previous year. Patients identified by the SBT as having possible cognitive impairment or dementia were excluded. Those findings were discussed with the clinical team and the patient so that appropriate follow-up could be provided. Those who passed the SBT then completed the GDS-15 and GAI. Patients could complete the measure on their own or by having questions read aloud. Oral administration of self-report assessments including the GAI has been conducted in previous studies [23]. The fellow took notes during the assessment about any comments made regarding the measures and confusion observed during administration. 
After completing the questionnaires, the patients were provided feedback regarding whether they had a positive screen for anxiety or depression and, if so, the effect that these symptoms might have on their functioning was discussed briefly. Patients were informed about several treatment options, and referrals were made with patients' consent. All assessments were completed in clinic rooms to ensure patient privacy.

\section{RESULTS}

\section{Feasibility of Assessment}

Of the 131 records reviewed, 56 patients were invited to participate in the project. Thirty-eight patients were excluded for the aforementioned reasons. The remaining 37 patients were not approached because of scheduling conflicts or concurrent assessments with other patients. Of the 56 patients approached, 3 (5.4\%) declined participation. The remaining 53 (94.6\%) patients agreed to participate in the project, which suggests that they were amenable to completing a brief anxiety assessment in primary care. Of the 53 patients, 3 obtained elevated scores on the SBT consistent with possible cognitive impairment or dementia and were thus excluded from the project. The mean score on the SBT $(1.14 \pm 1.51)$ fell below the SBT cutpoint of $>4$ for possible cognitive impairment or dementia. The Table contains demographic characteristics for the 50 patients who completed the GAI and then the GDS-15.

Interestingly, the majority of patients $(80 \%, n=40)$ elected to have the psychology fellow read the questions aloud. Although reading the questions aloud is more timeconsuming, this process can allow an assessor to gather other information that could be used in making recommendations to the patient at the end of the assessment. Based on observations from the interviews, more clarification was needed for the GDS-15 questions than for the GAI questions. The fellow repeated the instructions and reminded the patients of the $1 \mathrm{wk}$ timeframe during the administration of the GDS-15 because patients often responded to the questions based on long-standing changes rather than changes that occurred within the past week. Three patients chose not to respond to item 15 on the GDS-15, "Do you think that most people are better off than you are" because they stated they could not make this judgment. On the GAI, two patients were unsure of the dif-
Table.

Patient characteristics and assessment findings.

\begin{tabular}{|c|c|}
\hline Characteristic & Value \\
\hline Age, yr (mean \pm SD) & $78.5 \pm 7.4$ \\
\hline Male & $50(100)$ \\
\hline \multicolumn{2}{|l|}{ Race/Ethnicity, $n$ (\%) } \\
\hline White, non-Latino & $41(82.0)$ \\
\hline White, Latino & $3(6.0)$ \\
\hline African American & $2(4.0)$ \\
\hline Asian American & $1(2.0)$ \\
\hline Other & $3(6.0)$ \\
\hline \multicolumn{2}{|l|}{ Brief Cognitive Assessment, $n(\%)$} \\
\hline Yes, Assessment Completed Within Past Year & $21(42.0)$ \\
\hline MMSE & $13(26.0)$ \\
\hline MOCA & $3(6.0)$ \\
\hline Mini-Cog & $5(10.0)$ \\
\hline No, SBT Administered & $29(58.0)$ \\
\hline \multicolumn{2}{|l|}{ Psychiatric Diagnoses on Problem List, ${ }^{*} n(\%)$} \\
\hline Alcohol Use Disorder & $1(2.0)$ \\
\hline Anxiety Disorder NOS & $5(10.0)$ \\
\hline Depressive Disorders (including dysthymia) & $7(14.0)$ \\
\hline Posttraumatic Stress Disorder & $1(2.0)$ \\
\hline \multicolumn{2}{|l|}{ Psychiatric Diagnoses on Assessments, $^{\dagger} n(\%)$} \\
\hline Anxiety (GAI > 8) & $2(4.0)$ \\
\hline Depression (GDS-15 > 6) & $3(6.0)$ \\
\hline SBT (mean \pm SD) & $1.14 \pm 1.51$ \\
\hline GAI (mean \pm SD) & $1.94 \pm 2.77$ \\
\hline GDS-15 (mean \pm SD) & $2.06 \pm 2.09$ \\
\hline
\end{tabular}

${ }^{*}$ Eleven patients had one or more psychiatric diagnoses. Comorbidities were anxiety and depression $(n=2)$ and depression and alcohol use $(n=1)$.

${ }^{\dagger}$ GAI detected one new anxiety case; GDS-15 detected one new depression case; other positive screens were for individuals with diagnoses already on their problem lists.

GAI = Geriatric Anxiety Inventory, GDS-15 = 15-item Geriatric Depression Scale, MMSE $=$ Mini-Mental State Examination, MOCA $=$ Montreal Cognitive Assessment, NOS $=$ not otherwise specified, SBT $=$ Short Blessed Test, $\mathrm{SD}=$ standard deviation.

ference between adjectives for frequency of symptoms, specifically the qualifiers of "often" and "sometimes."

We measured time spent completing the GAI, GDS15 , and a brief cognitive assessment (if administered during the QI project) and discussing the results for the last 18 patients interviewed based on a recommendation made part way through the project by a team member. Of the 18 patients, 10 had completed a brief cognitive assessment within the past year and were only administered the GAI and GDS-15, whereas the 8 remaining participants 
were administered the SBT, GAI, and GDS-15. Administration and feedback lasted $6.20 \pm 2.53 \mathrm{~min}$ for the GAI and GDS-15 $(n=10)$ and $13.88 \pm 4.49 \mathrm{~min}$ for the SBT, GAI, and GDS-15 $(n=8)$. The administration of the SBT almost doubled the duration of the interaction, but there was no evidence that the SBT administration led to patients being frustrated or inattentive when completing the self-report measures. Post hoc analyses demonstrated that GAI and GDS-15 scores did not differ for patients who completed cognitive assessments compared with patients who did not complete cognitive assessments.

\section{Anxiety and Depression Outcomes}

Although the mean scores of the sample were low for the GAI and GDS-15 (Table), 90.0 percent of GAI items and 93.3 percent of GDS-15 items were endorsed by at least one Veteran in the clinic, with several items being frequently endorsed. The range of scores for the GAI was 0 to 10 , and was 0 to 9 for the GDS-15. The three most endorsed items on the GAI were as follows: "I worry a lot of the time" (22\%), "My own thoughts often make me anxious" (22\%), and "Little things bother me a lot" (20\%). The three most endorsed items on the GDS-15 were: "Do you prefer to stay at home rather than going out and doing new things?” (yes, 36\%), "Do you feel full of energy?" (no, 36\%), and "Have you dropped many of your activities and interests?” (yes, 28\%). A moderate association between GAI and GDS-15 total scores was also found ( $r=0.58, p<0.001)$.

Findings from the chart review demonstrated that $11(22.0 \%)$ of the older primary care patients who participated in the project had at least one psychiatric diagnosis recorded on their problem list. Patients with one or more psychiatric diagnoses obtained significantly higher scores on the GAI (mean $=4.73 \pm 1.15$ ) compared with patients without psychiatric diagnoses (mean $=1.15 \pm 1.86$, $t(11.46)=-3.10, p=0.01)$. Patients with one or more psychiatric diagnoses did not have significantly higher scores on the GDS-15 (mean $=3.18 \pm 2.56$ ) compared with those patients without psychiatric diagnoses (mean $=1.74 \pm$ 1.86, $t(13.12)=-1.74, p=0.11)$.

Because the GAI and GDS-15 were both employed as screens for anxiety and depression, we report on the number of positive screens for either anxiety or depression and whether these individuals were newly identified. Using a cutpoint of $>8$ on the GAI, two patients were identified as having a potential anxiety disorder. Both patients had psychiatric disorders listed on their problem list, but only one had a previous diagnosis of an anxiety disorder. A post hoc examination of response to the five GAI items that appear on the GAI short-form (GAI-SF) [33] revealed that the same two patients were the only two identified as having a potential anxiety disorder. Three patients were identified as having a depressive disorder using a cutpoint of $>6$ on the GDS-15. Two of the three patients already had depressive disorders on their problem list. One patient who screened positively for depression also screened positively for anxiety. Overall, four patients obtained positive depression or anxiety screens or both. All patients were provided with information about VA mental and behavioral health referrals and other available resources (e.g., My HealtheVet stress management modules). One patient accepted a referral for individual psychotherapy via a Geriatric Primary Care Behavioral Health program [34]. The clinical nurse specialist in the clinic opted to case manage the patient with both anxiety and depression. The two remaining patients were not interested in psychotherapy but received individualized behavioral recommendations.

There were nine instances in which patients with either anxiety or depression diagnoses on their problem lists were not identified as having a disorder using the GAI or GDS-15 screen. Many of the patients with psychiatric problems already listed on their problem lists were currently being treated for those disorders as well. Specifically, four patients who had anxiety diagnoses listed as active problems in their medical record did not have scores $>8$ on the GAI, and five patients with depression diagnoses on problem lists did not have scores $>6$ on the GDS- 15 .

\section{Implementation Findings}

Following the examination of the feasibility of the GAI in the geriatric primary care clinic, the measure has been expanded for use by two clinical nurse specialists, a licensed clinical social worker, a psychologist, and psychology trainees in five clinics in our healthcare system: two geriatric interdisciplinary primary care, a geriatric outpatient consultation, a geriatric primary care behavioral health, and a clinical video telehealth geriatric consultation. Providers elect to administer the GAI to patients whom they suspect may be experiencing anxiety or worries. The incorporation of the GAI as one available assessment represents a change in clinical practice. Following this QI project, the psychology fellow discussed the QI project findings during monthly clinical team meetings and disseminated the results during a seminar presentation on the assessment and treatment of late-life anxiety. Many 
providers in the Geriatric Primary Care Behavioral Health program began to use the GAI to measure anxiety treatment outcomes instead of measures not specifically designed for older adults, such as the GAD interview in the Mini-International Neuropsychiatric Interview [35] or the BAI. Finally, as an implementation outcome, patients with anxiety symptoms in the geriatric primary care clinic are now actively followed (i.e., assessed annually or more frequently) using the GAI and further assessed or referred for treatment when anxiety symptoms on the GAI crest the cutpoint of 8 .

\section{DISCUSSION}

We examined the feasibility of incorporating an anxiety screen, the GAI, into clinical care in a geriatrics primary care clinic. The main goal of the QI project was to determine whether a geriatric screening measure provided useful information to detect anxiety and improve clinic procedures in a primary care clinic serving older patients. Discussion of the new clinical procedure, the percentage of clinic patients with anxiety and depression, and implementation findings follow.

Using the cutpoint of $>8$ on the GAI, as recommended in previous studies [23], two patients (4.0\%) were identified as having a possible anxiety disorder. Although both patients had other Axis I disorders, only one of the two had a previous diagnosis of anxiety. The screening only detected one of five individuals ( $10 \%$ of sample) who had an anxiety disorder according to the medical record. As noted earlier, some patients with diagnoses on their problem lists were already receiving treatment for these disorders, which may have led to full or partial remission of the anxiety disorders. These preliminary findings suggest that anxiety was not being substantially underdiagnosed in our geriatric primary care clinic. Additionally, the overall low percentage of patients with anxiety disorders could be due to the composition of the clinic since most patients are World War II or Korean war era Veterans. Higher rates of anxiety and depression disorders would be expected if the sample included more Vietnam war era Veterans [3]. The finding that the patients with psychiatric disorders on their problem lists obtained higher scores on the GAI than patients without psychiatric disorders suggests that the GAI may be more sensitive to nonspecific distress associated with psychiatric symptoms than the GDS-15. Further assessment with a diagnostic interview for anxiety disorders is needed to disentangle this issue. These preliminary findings suggest that the GAI may provide an incremental improvement in the detection of psychiatric distress beyond standard VHA assessments included in a primary care visit.

Overall, we found good patient acceptability of both the GAI and GDS-15, with 94.6 percent of patients agreeing to the anxiety assessment and 100 percent completing the anxiety assessment once it was initiated. Interestingly, the majority of patients elected to have the questions on the GAI and GDS-15 read aloud. Vision difficulties may have played a role in the choice to have clinicians read the questions aloud, but this was not documented. Additionally, it seemed that patients valued the interaction with a clinician, as evidenced by the patients who provided information to the clinician beyond the dichotomous response to the questions. Administration and feedback following completion of a depression and anxiety screen was brief, taking approximately $6.5 \mathrm{~min}$; however, when a brief cognitive assessment was included, the time spent with the patient doubled. The increased time was likely related to the administration time for the SBT (approximately 5 min or less) and additional time needed to answer questions related to subjective cognitive complaints or ways to maintain one's cognitive abilities. Benefits of oral administration of the GAI and GDS-15 include the opportunity to clarify the self-report measures' instructions. For instance, patients are asked to respond to GDS-15 questions based on the past week, but often patients respond to the questions about how they are feeling generally, which leads to the need for further differentiating of whether patients were experiencing a depressive episode or adjusting to living with a chronic illness or both. Also, reading the GAI and GDS-15 questions aloud provides an opportunity for patients to discuss their symptoms in further detail. This individualized information was used to make patient-centered recommendations following positive anxiety or depression screens. Two of the four patients with positive anxiety (GAI) or depression (GDS-15) screens received additional clinical services (psychotherapy, case management), and all four patients with positive screens on the GAI or GDS-15 received psychoeducation and individualized recommendations. A considerable drawback to this process is the staff time needed to administer the screens and patient time needed to complete the assessments. Our findings suggest that implementing an anxiety screen is feasible and may improve patient care. Following our test of the feasibility of screening for anxiety, we found that providers from multiple 
disciplines adopted this measure in their practice to both screen for and monitor anxiety. We observed increased use of this measure in several clinics, as evidenced by providers requesting copies of the GAI measure without much prompting by project staff.

Whereas the percentages of patients with anxiety (4.0\%) and depression (6.0\%) as identified by the GAI and GDS-15 were relatively low compared with the percentages of patients with those disorders per medical record review (10\% and $14 \%$, respectively), several patients endorsed having symptoms of anxiety and depression. Yet these symptoms fell below the threshold cutpoints. On the GAI, approximately one in five patients reported worrying a lot of the time, having anxiety because of their own thoughts, and being bothered by little things. On the GDS15 , one in three patients indicated that they preferred to stay at home and one in four patients reported dropping their interests or activities. These elevated levels of symptoms warrant examination in a future study because elevated anxiety symptoms, not necessarily meeting criteria for an anxiety disorder, are associated with declines in functioning [7].

Among older adults, anxiety and depression often cooccur [22]. The finding of a large association of GAI and GDS-15 scores aligns with findings of a high degree of comorbidity of anxiety and depression in older adults, although this may raise concerns regarding value added for anxiety assessment. Our findings suggest, however, that the presence of both anxiety and depression indicates a higher degree of psychological distress in older primary care patients, which is consistent with other research [36]. Although treatments for depression and anxiety have a high degree of overlap, differentiating anxiety and depression symptoms is essential to patient-centered care. One way to address the large association between anxiety and depression is to carefully select a measure with less overlap with depression. Diefenbach et al. report that the 5-item GAI-SF has better discriminant validity with GDS than the 20-item GAI [20]. The GAI-SF also may reduce burden on staff and patients if used in future clinical assessments. Following positive findings on the GAI-SF, additional assessment could take place, which could include administration of the remaining 15 GAI items. Taken together, our findings suggest that administering a brief anxiety screen that has less overlap with depression (e.g., GAI-SF) would help identify anxiety symptoms and would provide more patient-centered care to Veterans.
Limitations to the present project include a relatively modest sample size, absence of a control group, and the exclusion of 27.4 percent of older Veterans due to known dementia or possible cognitive impairment or dementia. Because the present project was part of clinical care and not a research study, no structured clinical interviews were used to identify the presence of anxiety and depressive disorders. The participants in the QI project were mostly white male Veterans. Thus, the findings do not necessarily generalize to other racial or ethnic minority Veterans or female Veterans. Despite these limitations, this project provides evidence of the utility of the GAI in a VA geriatric primary care clinic. After the feasibility of administering the GAI was determined, several providers from multiple disciplines incorporated this measure into their clinical practice.

\section{CONCLUSIONS}

Our findings suggest that the GAI is appropriate as a tool for interdisciplinary providers in VA primary care clinics to screen for and monitor anxiety symptoms in older patients. Yet, the GAI does not appear to be able to differentiate anxiety symptoms from depression. Detection of anxiety likely benefits patients by identifying distressing symptoms and thereby initiating conversations about available treatments with their providers. Future evaluations should examine the GAI-SF in comparison with the longer form in a large clinical sample of older Veterans to determine whether the brief form may improve the detection of anxiety in the presence of depression.

\section{ACKNOWLEDGMENTS}

\section{Author Contributions:}

Study design: C. E. Gould, S. A. Beaudreau, G. Gullickson, J. L. Tenover, J. W. T. Huh.

Data collection and analysis: C. E. Gould.

Drafting of manuscript: C. E. Gould.

Critical revision of manuscript for important intellectual content:

C. E. Gould, S. A. Beaudreau, G. Gullickson, J. L. Tenover, J. W. T. Huh, E. A. Bauer.

Financial Disclosures: The authors have declared that no competing interests exist.

Funding/Support: This material was based on work supported by resources and use of facilities at the VA Palo Alto Health Care System. The first author was supported by a VA Advanced Fellowship in Geriatrics, VA Office of Academic Affairs, at the time of the project. 
Institutional Review: The VA Office of Research and Development reviewed the project prior to its implementation and granted it exempt status as a QI program.

Participant Follow-Up: The authors do not plan to inform participants of the publication of this study.

Disclaimer: The views expressed in this article are those of the authors and not necessarily those of the VA or the Federal Government.

\section{REFERENCES}

1. Gum AM, King-Kallimanis B, Kohn R. Prevalence of mood, anxiety, and substance-abuse disorders for older Americans in the national comorbidity survey-replication. Am J Geriatr Psychiatry. 2009;17(9):769-81. [PMID:19700949] http://dx.doi.org/10.1097/JGP.0b013e3181ad4f5a

2. Jordan BK, Schlenger WE, Hough R, Kulka RA, Weiss D, Fairbank JA, Marmar CR. Lifetime and current prevalence of specific psychiatric disorders among Vietnam veterans and controls. Arch Gen Psychiatry. 1991;48(3):207-15. [PMID:1996916] http://dx.doi.org/10.1001/archpsyc.1991.01810270019002

3. Gould CE, Rideaux T, Spira AP, Beaudreau SA. Depression and anxiety symptoms in male veterans and non-veterans: The Health and Retirement Study. Int J Geriatr Psychiatry. 2015;30(6):623-30. [PMID:25145943]

http://dx.doi.org/10.1002/gps.4193

4. Scherrer JF, Chrusciel T, Zeringue A, Garfield LD, Hauptman PJ, Lustman PJ, Freedland KE, Carney RM, Bucholz KK, Owen R, True WR. Anxiety disorders increase risk for incident myocardial infarction in depressed and nondepressed Veterans Administration patients. Am Heart J. 2010;159(5): 772-79. [PMID:20435185] http://dx.doi.org/10.1016/j.ahj.2010.02.033

5. Beaudreau SA, O’Hara R. Late-life anxiety and cognitive impairment: A review. Am J Geriatr Psychiatry. 2008;16(10): 790-803. [PMID:18827225] http://dx.doi.org/10.1097/JGP.0b013e31817945c3

6. Lenze EJ, Mulsant BH, Shear MK, Schulberg HC, Dew MA, Begley AE, Pollock BG, Reynolds CF 3rd. Comorbid anxiety disorders in depressed elderly patients. Am J Psychiatry. 2000;157(5):722-28. [PMID:10784464] http://dx.doi.org/10.1176/appi.ajp.157.5.722

7. Brenes GA, Guralnik JM, Williamson J, Fried LP, Penninx BW. Correlates of anxiety symptoms in physically disabled older women. Am J Geriatr Psychiatry. 2005;13(1):15-22. [PMID:15653936] http://dx.doi.org/10.1097/00019442-200501000-00004

8. Institute of Medicine. The mental health and substance use workforce for older adults: In whose hands? Washington (DC): Institute of Medicine; 2012. Available from: http://iom.nationalacademies.org/Reports/2012/The-MentalHealth-and-Substance-Use-Workforce-for-Older-Adults.aspx
9. Calleo J, Stanley MA, Greisinger A, Wehmanen O, Johnson M, Novy D, Wilson N, Kunik M. Generalized anxiety disorder in older medical patients: Diagnostic recognition, mental health management and service utilization. J Clin Psychol Med Settings. 2009;16(2):178-85. [PMID:19152056] http://dx.doi.org/10.1007/s10880-008-9144-5

10. Gonçalves DC, Byrne GJ. Interventions for generalized anxiety disorder in older adults: Systematic review and metaanalysis. J Anxiety Disord. 2012;26(1):1-11.

[PMID:21907538]

http://dx.doi.org/10.1016/j.janxdis.2011.08.010

11. Stanley MA, Wilson NL, Novy DM, Rhoades HM, Wagener PD, Greisinger AJ, Cully JA, Kunik ME. Cognitive behavior therapy for generalized anxiety disorder among older adults in primary care: A randomized clinical trial. JAMA. 2009;301(14):1460-67. [PMID:19351943] http://dx.doi.org/10.1001/jama.2009.458

12. Wetherell JL, Petkus AJ, White KS, Nguyen H, Kornblith S, Andreescu C, Zisook S, Lenze EJ. Antidepressant medication augmented with cognitive-behavioral therapy for generalized anxiety disorder in older adults. Am J Psychiatry. 2013;170(7):782-89. [PMID:23680817] http://dx.doi.org/10.1176/appi.ajp.2013.12081104

13. Gould CE, Beaudreau SA, Huh JW. A Veterans Health Administration imperative: Recommendations for detecting anxiety in older adults. Fed Pract. 2013;30(8):35-42.

14. Benjamin S, Herr NR, McDuffie J, Nagi A, Williams JW Jr. Performance characteristics of self-report instruments for diagnosing generalized anxiety and panic disorders in primary care: A systematic review. Washington (DC): Department of Veterans Affairs; 2011.

15. Spitzer RL, Kroenke K, Williams JB, Löwe B. A brief measure for assessing generalized anxiety disorder: The GAD-7. Arch Intern Med. 2006;166(10):1092-97. [PMID:16717171] http://dx.doi.org/10.1001/archinte.166.10.1092

16. Substance Abuse and Mental Health Services Administration. Older Americans behavioral health. Issue Brief 6: Depression and anxiety: Screening and intervention. Rockville (MD): SAMHSA; 2013.

17. Gould CE, Segal DL, Yochim BP, Pachana NA, Byrne GJ, Beaudreau SA. Measuring anxiety in late life: A psychometric examination of the geriatric anxiety inventory and geriatric anxiety scale. J Anxiety Disord. 2014;28(8):804-11.

[PMID:25271176]

http://dx.doi.org/10.1016/j.janxdis.2014.08.001

18. Pachana NA, Byrne GJ, Siddle H, Koloski N, Harley E, Arnold E. Development and validation of the Geriatric Anxiety Inventory. Int Psychogeriatr. 2007;19(1):103-14. [PMID:16805925] http://dx.doi.org/10.1017/S1041610206003504 
19. Pachana NA, Byrne GJ. The Geriatric Anxiety Inventory: International use and future directions. Austral Psychol. 2012;47(1):33-38.

20. Diefenbach GJ, Bragdon LB, Blank K. Geriatric anxiety inventory: Factor structure and associations with cognitive status. Am J Geriatr Psychiatry. 2014;22(12):1418-26. [PMID:23954040] http://dx.doi.org/10.1016/j.jagp.2013.04.014

21. Yesavage JA, Sheikh JI. Geriatric Depression Scale (GDS) recent evidence and development of a shorter violence. Clin Gerontol. 1986;5:165-73. http://dx.doi.org/10.1300/J018v05n01 09

22. Byers AL, Yaffe K, Covinsky KE, Friedman MB, Bruce ML. High occurrence of mood and anxiety disorders among older adults: The National Comorbidity Survey Replication. Arch Gen Psychiatry. 2010;67(5):489-96. [PMID:20439830] http://dx.doi.org/10.1001/archgenpsychiatry.2010.35

23. Diefenbach GJ, Tolin DF, Meunier SA, Gilliam CM. Assessment of anxiety in older home care recipients. Gerontologist. 2009;49(2):141-53. [PMID:19363010] http://dx.doi.org/10.1093/geront/gnp019

24. Yochim BP, Mueller AE, June A, Segal DL. Psychometric properties of the Geriatric Anxiety Scale: Comparison to the Beck Anxiety Inventory and Geriatric Anxiety Inventory. Clin Gerontol. 2010;34:21-33. http://dx.doi.org/10.1080/07317115.2011.524600

25. Kørner A, Lauritzen L, Abelskov K, Gulmann N, Marie Brodersen A, Wedervang-Jensen T, Marie Kjeldgaard K. The Geriatric Depression Scale and the Cornell Scale for Depression in Dementia. A validity study. Nord J Psychiatry. 2006;60(5):360-64. [PMID:17050293] http://dx.doi.org/10.1080/08039480600937066

26. Katzman R, Brown T, Fuld P, Peck A, Schechter R, Schimmel $\mathrm{H}$. Validation of a short Orientation-Memory-Concentration Test of cognitive impairment. Am J Psychiatry. 1983;140(6): 734-39. [PMID:6846631] http://dx.doi.org/10.1176/ajp.140.6.734

27. Blessed G, Tomlinson BE, Roth M. The association between quantitative measures of dementia and of senile change in the cerebral grey matter of elderly subjects. Br J Psychiatry. 1968;114(512):797-811. [PMID:5662937] http://dx.doi.org/10.1192/bjp.114.512.797

28. Morris JC, Heyman A, Mohs RC, Hughes JP, van Belle G, Fillenbaum G, Mellits ED, Clark C. The Consortium to Establish a Registry for Alzheimer's Disease (CERAD). Part I. Clinical and neuropsychological assessment of Alzheimer's disease. Neurology. 1989;39(9):1159-65. [PMID:2771064] http://dx.doi.org/10.1212/WNL.39.9.1159

29. Folstein MF, Folstein SE, McHugh PR. "Mini-mental state". A practical method for grading the cognitive state of patients for the clinician. J Psychiatr Res. 1975;12(3):189-98. [PMID:1202204] http://dx.doi.org/10.1016/0022-3956(75)90026-6
30. Nasreddine ZS, Phillips NA, Bédirian V, Charbonneau S, Whitehead V, Collin I, Cummings JL, Chertkow H. The Montreal Cognitive Assessment, MoCA: A brief screening tool for mild cognitive impairment. J Am Geriatr Soc. 2005; 53(4):695-99. [PMID:15817019] http://dx.doi.org/10.1111/j.1532-5415.2005.53221.x

31. Ott BR, Fogel BS. Measurement of depression in dementia: Self vs. clinician rating. Int J Geriatr Psychiatry. 1992;7: 899-904. http://dx.doi.org/10.1002/gps.930071209

32. Borson S, Scanlan JM, Chen P, Ganguli M. The Mini-Cog as a screen for dementia: Validation in a population-based sample. J Am Geriatr Soc. 2003;51(10):1451-54. [PMID:14511167] http://dx.doi.org/10.1046/j.1532-5415.2003.51465.x

33. Byrne GJ, Pachana NA. Development and validation of a short form of the Geriatric Anxiety Inventory - the GAI-SF. In Psychogeriatr. 2011;23(1):125-31. [PMID:20561386]

34. Huh JW, Woodhead E, Brunskill S, Gould CE, McConnell $\mathrm{K}$, Tenover JT. Geriatric mood management program development and evaluation: A Geriatric Research Education and Clinical Center (GRECC) clinical demonstration project. Fed Pract. 2014;31(5):32-35.

35. Sheehan DV, Lecrubier Y, Sheehan KH, Amorim P, Janavs J, Weiller E, Hergueta T, Baker R, Dunbar GC. The MiniInternational Neuropsychiatric Interview (M.I.N.I.): The development and validation of a structured diagnostic psychiatric interview for DSM-IV and ICD-10. J Clin Psychiatry. 1998; 59(Suppl 20):22-33, quiz 34-57.

[PMID:9881538]

36. Watts SC, Bhutani GE, Stout IH, Ducker GM, Cleator PJ, McGarry J, Day M. Mental health in older adult recipients of primary care services: Is depression the key issue? Identification, treatment and the general practitioner. Int J Geriatr Psychiatry. 2002;17(5):427-37. [PMID:11994931] http://dx.doi.org/10.1002/gps.632

Submitted for publication October 24, 2014. Accepted in revised form May 15, 2015.

This article and any supplementary material should be cited as follows:

Gould CE, Beaudreau SA, Gullickson G, Tenover JL, Bauer EA, Huh JW. Implementation of a brief anxiety assessment and evaluation in a Department of Veterans Affairs geriatric primary care clinic. J Rehabil Res Dev. 2016;53(3):335-44.

http://dx.doi.org/10.1682/JRRD.2014.10.0258

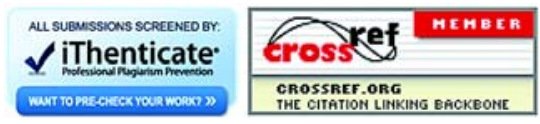


